Preprint typeset in JHEP style - HYPER VERSION

\title{
How many black holes fit on the head of a pin?*
}

\author{
Frederik Denef $^{1}$ and Gregory W. Moore ${ }^{2}$ \\ ${ }^{1}$ Instituut voor Theoretische Fysica, KU Leuven, \\ Celestijnenlaan 200D, B-3001 Leuven, Belgium \\ 2 NHETC and Department of Physics and Astronomy, Rutgers University, \\ Piscataway, NJ 08855-0849, USA \\ frederik.denef@fys.kuleuven.be, gmoore@physics.rutgers.edu
}

\begin{abstract}
The Bekenstein-Hawking entropy of certain black holes can be computed microscopically in string theory by mapping the elusive problem of counting microstates of a strongly gravitating black hole to the tractable problem of counting microstates of a weakly coupled D-brane system, which has no event horizon, and indeed comfortably fits on the head of a pin. We show here that, contrary to widely held beliefs, the entropy of spherically symmetric black holes can easily be dwarfed by that of stationary multi-blackhole "molecules" of the same total charge and energy. Thus, the corresponding pin-sized D-brane systems do not even approximately count the microstates of a single black hole, but rather those of a zoo of entropically dominant multicentered configurations.
\end{abstract}

\footnotetext{
${ }^{*}$ Fourth prize in the Gravity Research Foundation Essay competition 2007.
} 
Explaining the microscopic origin of the entropy of black holes has been a longstanding problem [1, 2]. Impressive progress was made, within the context of string theory, by Strominger and Vafa, who accounted for the entropy of certain supersymmetric five dimensional charged black holes in terms of D-brane microstates [3]. This was extended to certain four dimensional supersymmetric black holes in 4,5 .

In this essay, we revisit some of the assumptions made in these derivations, in particular the crucial and rather stunning assertion that by tuning the string coupling to zero one can fit a black hole, without loss of entropy, on the head of a pin. We do not dispute this fact - on the contrary, we will show that quite a bit more fits on that pin, namely supersymmetric multi-centered black hole bound states, and we will demonstrate that these can actually dwarf the entropy of single centered black holes. Although we do not take the titular question as seriously as the medieval scholastics allegedly took theirs [6], the answer has some tangible implications for the program of reproducing statistically the entropy of black holes.

We will focus on black holes in four dimensional $\mathcal{N}=2$ supersymmetric theories. The basic idea underlying the microscopic entropy computation is simple. The weighted number of supersymmetric one-particle states with a given conserved charge $\Gamma$ is given by the Witten index

$$
\Omega\left(\Gamma ; \tau_{\infty}\right)=\operatorname{Tr}_{\Gamma}(-1)^{2 J_{3}}\left(J_{3}\right)^{2} e^{-\beta H},
$$

where $J_{3}$ is the 3 -component of the angular momentum, $H$ is the energy above the supersymmetric lower bound, the trace is over the subsector of the Hilbert space of states with charge $\Gamma$, and $\tau_{\infty}$ denotes the values at spatial infinity of certain scalars parametrizing the vacua of the theory. The index has the remarkable property of being independent of $\beta$ and other couplings of the theory since nonsupersymmetric (i.e. $H>0$ ) states cancel in bose-fermi pairs, allowing it to be computed exactly in a suitably controlled regime [7].

Now, in four dimensional string compactifications, in the limit of weak coupling and small internal metric curvatures, the Hilbert space of charged supersymmetric one particle ground states is well understood: it is given by quantizing the moduli space of supersymmetric configurations of D-branes wrapping various cycles of the Calabi-Yau compactification manifold $X$, and sitting at a single point in the noncompact spacetime - that point being the titular pinhead. On the other hand, the four dimensional low energy effective supergravity theory has supersymmetric black hole solutions carrying identical charges. Identifying these as the classical strong gravitational coupling description of the weakly coupled D-brane states and exploiting the deformation invariance of the index, one thus obtains an indirect way to compute the microcanonical statistical entropy of a black hole by counting the corresponding supersymmetric D-brane ground states [3].

Although this approach has been spectacularly successful in many cases, leading to exact quantitative agreement with the macroscopic Bekenstein-Hawking formula for the entropy, one could raise several objections. We focus on one of them, which we believe is by far the most significant one, in light of results we recently reported in [\$].

The problem originates in the fact that the trace (11) runs over all one particle states of charge $\Gamma$, not just those corresponding to spherically symmetric black holes. For example, 
there could be additional particles orbiting the black hole, or entire galaxies for that matter - any state with total charge $\Gamma$ should a priori be included. Hence one could wonder if the identification of the index with the ground state degeneracy of a single, spherically symmetric black hole is really justified.

This objection is usually not considered to be serious, since orbiting galaxies, being non-supersymmetric, just drop out of the trace (1). Only supersymmetric configurations

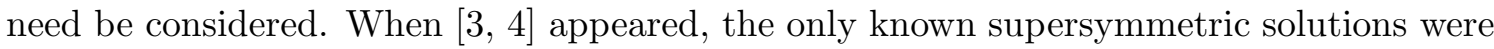
spherically symmetric black holes [9, 10]. Moreover, from known results about black hole physics, it seemed intuitively obvious that the most entropic minimal energy supergravity solution should be a single black hole. It thus made perfect sense to identify the leading D-brane entropy with the leading single centered black hole entropy.

Subsequently, however, more general supersymmetric solutions were discovered - stationary, multicentered, "molecular" black hole bound states [11, 12, 13, 14]. These are completely determined by harmonic functions

$$
H^{\Lambda}(\vec{x}):=\sum_{i} \frac{p_{i}^{\Lambda}}{\left|\vec{x}-\vec{x}_{i}\right|}+h^{\Lambda}, \quad H_{\Lambda}(\vec{x}):=\sum_{i} \frac{q_{i, \Lambda}}{\left|\vec{x}-\vec{x}_{i}\right|}+h_{\Lambda},
$$

where $\vec{x}_{i}$ is the position of the $i$-th center, $\Gamma_{i}:=\left(p_{i}^{\Lambda}, q_{\Lambda, i}\right)$ its (magnetic, electric) charges with respect to the $U(1)^{n+1}$ gauge group labeled by $\Lambda=0, \ldots, n,\left(h^{\Lambda}, h_{\Lambda}\right)$ are constants determined by the total charge $\Gamma=\sum_{i} \Gamma_{i}$ and $\tau_{\infty}$, and we work in units with Newton's constant $G_{N} \equiv 1$.

Remarkably, given $H(\vec{x})$, the explicit solution for all fields can be obtained from one "master" function $S$ defined on the $2 n+2$ dimensional charge space and scaling as $S(\lambda \Gamma)=$ $\lambda^{2} S(\Gamma)$ [14]. In particular, the metric is

$$
d s^{2}=-\frac{\pi}{S(H)}(d t+\omega)^{2}+\frac{S(H)}{\pi} d \vec{x}^{2}
$$

where $\omega=\omega_{i}(\vec{x}) d x^{i}$ solves

$$
d \omega=\langle * d H, H\rangle
$$

with $\langle A, B\rangle:=A^{\Lambda} B_{\Lambda}-A_{\Lambda} B^{\Lambda}$ the duality invariant product and $*$ the Hodge star on $\mathbb{R}^{3}$.

The scaling homogeneity of $S$ together with (3) implies that the area of the horizon of the $i$-th center at $\vec{x}=\vec{x}_{i}$ is given by $A_{i}=4 S\left(\Gamma_{i}\right)$, and hence the corresponding BekensteinHawking entropy equals $S\left(\Gamma_{i}\right)$. Therefore $S$ is the single centered supersymmetric entropy function, which in turn is completely determined by the topological data of the string compactification. In order for the solution to exist, $H(\vec{x})$ must remain within the domain where the function $S$ is positive.

Equation (4) can be solved provided the integrability condition $\langle\Delta H, H\rangle=0$ is satisfied. Since $\Delta H \sim \sum_{i} \delta^{3}\left(\vec{x}-\vec{x}_{i}\right) \Gamma_{i}$, the $\vec{x}_{i}$ are constrained and hence these configurations are typically genuine bound states, in the sense that one cannot move the centers away from each other without input of energy. For example for two centered solutions one gets the constraint

$$
\left|\vec{x}_{1}-\vec{x}_{2}\right|=-\frac{\left\langle\Gamma_{1}, \Gamma_{2}\right\rangle}{\left\langle\Gamma_{1}, h\right\rangle} .
$$


Since $h$ depends on $\tau_{\infty}$ and the right hand side must be positive, the existence of these black hole bound states depends on the choice of vacuum.

Thus, we face a problem: the index (1) receives nonvanishing contributions not just from a single black hole, but, in general, also from a disturbingly complex zoo of multicentered black hole bound states with the same total charge $\Gamma$. All of these collapse to a single D-brane in the weak string coupling limit [15]. This answers the titular question definitively as "Many, many, ..." but leaves us wondering how to extract the actual single centered black hole entropy from (11).

It has generally been assumed that a single centered black hole dominates the entropy of its charge sector, so the existence of multicentered configurations is merely a minor nuisance, completely negligible in the thermodynamic limit. Indeed when several black holes are dynamically merged, the second law of thermodynamics implies that their horizon entropy increases. However, for our "molecular" bound states, merging the centers requires adding enough energy to the system to overcome potential barriers, necessarily producing a nonextremal final black hole. The possibility remains that multicentered extremal solutions might be more entropic than the corresponding single centered extremal hole.

One of the surprising results reported in 8 is that in fact, in suitable parameter regimes, multicentered entropy does dominate single centered entropy in the uniform large charge limit. More precisely, when charges $\Gamma$ obtained by wrapping D4, D2 and D0 branes around various cycles of $X$ are scaled up as $\Gamma \rightarrow \Lambda \Gamma$, there exist two centered solutions with horizon entropy scaling as $\Lambda^{3}$ while the single centered entropy scales as $\Lambda^{2}$.

Let us give a concrete example of this phenomenon, referring to 8 for more details. Consider type IIA string theory compactified on $X=T_{1}^{2} \times T_{2}^{2} \times T_{3}^{2}$, a product of three two-tori. Let $D$ be the 4 -cycle $\left(T_{1}^{2} \times T_{2}^{2}\right)+\left(T_{2}^{2} \times T_{3}^{2}\right)+\left(T_{3}^{2} \times T_{1}^{2}\right)$ and let $\tilde{D}$ be the 2 -cycle $T_{1}^{2}+T_{2}^{2}+T_{3}^{2}$. Then the entropy function of a charge $\Gamma$ corresponding to $p^{0} \mathrm{D} 6$-branes on $X, p$ D4-branes on $D, q$ D2-branes on $\tilde{D}$ and $q_{0}$ D0-branes is given by

$$
S(\Gamma)=\pi \sqrt{-4 p^{3} q_{0}+3 p^{2} q^{2}+6 p^{0} p q q_{0}-4 p^{0} q^{3}-\left(p^{0} q_{0}\right)^{2}} .
$$

Now consider a total charge

$$
\Gamma=\left(p^{0}, p, q, q_{0}\right)=\Lambda(0,6,0,-12),
$$

in a background in which the area of each $T^{2}$ equals $v$ (which plays the role of $\tau_{\infty}$ ). Then for any $v$, there exists a single centered solution with horizon entropy given by (6):

$$
S_{1}=72 \sqrt{2} \pi \Lambda^{2}
$$

However, when $v>\sqrt{18} \Lambda$, there is also a two-centered bound state with charges

$$
\Gamma_{1}=\left(1,3 \Lambda, 6 \Lambda^{2},-6 \Lambda\right), \quad \Gamma_{2}=\left(-1,3 \Lambda,-6 \Lambda^{2},-6 \Lambda\right)
$$

The constant terms in the harmonic functions (2) are $h=\left(0, \frac{1}{\sqrt{2 v}}, 0,-\sqrt{\frac{v^{3}}{2}}\right)$, and the equilibrium separation (5) is $\left|\vec{x}_{1}-\vec{x}_{2}\right|=12 \sqrt{2} \Lambda\left(9 \Lambda^{2}-1\right) \sqrt{v} /\left(v^{2}-18 \Lambda^{2}\right)$. The resulting 
metric is well defined, with $S(H)$ real and positive everywhere (see fig. 1). The two centers have equal horizon entropy, summing up to a total entropy

$$
S_{2}=12 \pi \Lambda \sqrt{3 \Lambda^{4}-1} \sim \Lambda^{3}
$$

which is indeed parametrically larger than the single centered entropy (8). (This does not contradict the holographic principle, since the area of any surface enclosing the black holes grows at least as fast as $\Lambda^{3}$.)

When $v$ is kept fixed while sending $\Lambda \rightarrow \infty$, (5) ceases to have a solution and hence these 2-centered solutions disappear from the spectrum. However, the regime in which (11) can be reliably computed microscopically with present technology is precisely the $v \rightarrow \infty$ limit, so we conclude that at large $\Lambda$, the usual weakly coupled, weakly curved wrapped D-branes are not computing the entropy of a single black hole, but rather that of complicated multicentered configurations. The fact that the entropy computations [3, 3,5$]$ matched so beautifully to the single black hole entropy is due to the "accident" that in the special charge regime in which the microscopic asymptotics could be extracted, there just happen to be no competing mul-

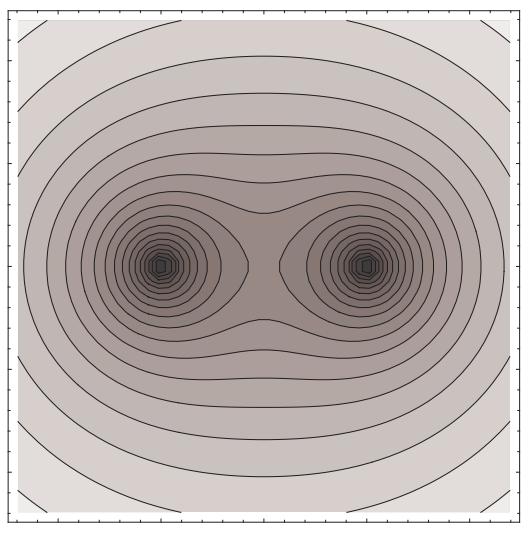

Figure 1: Metric warp factor $\pi / S(H)$ for the 2-centered example. ticentered solutions. The microscopic counting in the regime where multi-centered solutions dominate has not yet been done.

In conclusion, our scholastic question raises a key issue: we need new ideas to compute microstates in the generic strong coupling regime. This is a fortiori true in the absence of supersymmetry.

\section{Acknowledgments}

This work was supported in part by the DOE under grant DE-FG02-96ER40949, by the Belgian Federal Office for Scientific, Technical and Cultural Affairs through the "Interuniversity Attraction Poles Programme - Belgian Science Policy" P5/27 and by the European Community's Human Potential Programme under contract MRTN-CT-2004-005104 "Constituents, fundamental forces and symmetries of the universe". FD would like to thank the Galileo Galilei Institute for Theoretical Physics for hospitality and the INFN for partial support during the completion of this work.

\section{References}

[1] R.M. Wald, "The thermodynamics of black holes," Living Rev. Relativity, 4 (2001), 6. http://relativity.livingreviews.org/Articles/lrr-2001-6/

[2] T. Damour, "The entropy of black holes: A primer," arXiv:hep-th/0401160. 
[3] A. Strominger and C. Vafa, "Microscopic Origin of the Bekenstein-Hawking Entropy," Phys. Lett. B 379 (1996) 99 [arXiv:hep-th/9601029].

[4] J. M. Maldacena, A. Strominger and E. Witten, "Black hole entropy in M-theory," JHEP 9712 (1997) 002 [arXiv:hep-th/9711053].

[5] C. Vafa, "Black holes and Calabi-Yau threefolds," Adv. Theor. Math. Phys. 2, 207 (1998) [arXiv:hep-th/9711067].

[6] T. Aquinas, "Summa theologiae I," q. 52 art. 3 (1274), http://www.newadvent.org/summa/105203.htm; see also http://plato.stanford.edu/entries/duns-scotus/.

[7] E. Witten, "Constraints On Supersymmetry Breaking," Nucl. Phys. B 202, 253 (1982).

[8] F. Denef and G. W. Moore, "Split states, entropy enigmas, holes and halos," arXiv:hep-th/0702146.

[9] S. Ferrara, R. Kallosh and A. Strominger, "N=2 extremal black holes," Phys. Rev. D 52 (1995) 5412 [arXiv:hep-th/9508072].

[10] A. Strominger, "Macroscopic Entropy of $N=2$ Extremal Black Holes," Phys. Lett. B 383 (1996) 39 [arXiv:hep-th/9602111].

[11] K. Behrndt, D. Lust and W. A. Sabra, "Stationary solutions of N = 2 supergravity," Nucl. Phys. B 510, 264 (1998) [arXiv:hep-th/9705169].

[12] F. Denef, "Supergravity flows and D-brane stability," JHEP 0008, 050 (2000) [arXiv:hep-th/0005049].

[13] G. Lopes Cardoso, B. de Wit, J. Kappeli and T. Mohaupt, "Stationary BPS solutions in N = 2 supergravity with $\mathrm{R}^{* * 2} 2$ interactions," JHEP 0012, 019 (2000) [arXiv:hep-th/0009234].

[14] B. Bates and F. Denef, "Exact solutions for supersymmetric stationary black hole composites," arXiv:hep-th/0304094.

[15] F. Denef, "Quantum quivers and Hall/hole halos," JHEP 0210, 023 (2002) [arXiv:hep-th/0206072]. 